\title{
EXPONENTIATION OF OPERATOR LIE ALGEBRAS ON BANACH SPACES 1
}

\author{
BY ROBERT T. MOORE
}

Communicated by S. Smale, April 7, 1965

1. Introduction. By a $C^{\infty}$ Lie algebra of operators on a Banach space $x$ we shall mean a pair $(g, D)$ consisting of (a) a dense linear subset ("domain") $D$ of the space $X$ and (b) a finite dimensional real vector space $g$ of operators $X, Y, Z \ldots$ defined on $D$ such that $X \mathscr{D C D}$ for all $X \in g$ (" $C^{\infty}$ condition") and such that the commutator Lie product $[X, Y]=X Y-Y X$ carries $g \times g$ into $g$.

It is well known that every strongly continuous representation $U$ of a Lie group $G$ on $X$ gives rise to a number of different domains $D$ of $C^{\infty}$ vectors for $U$ on which the Lie algebra $g$ of $G$ may be represented to give such a $C^{\infty}$ Lie algebra $(g, D)$ (cf. Segal [10], Gårding [4], Harish-Chandra [5], Cartier and Dixmier [2] and Nelson [8]). Here $U(G)$ is a generalized exponential of $(g, D)$. Therefore we will call a $C^{\infty}$ Lie algebra of operators exponentiable in case the simply connected Lie group $G$ whose Lie algebra is isomorphic with $g$ has a strongly continuous representation $U$ on $X$ such that when $f \in D$ :

$$
\lim _{t \rightarrow 0} t^{-1}[U(\exp t X) f-f]=X f
$$

(here we have identified $(g, D)$ with the Lie algebra of $G$ ). We will discuss the question: When is a $C^{\infty}$ Lie algebra of operators on $D$ exponentiable? Nelson [8] gives a sufficient condition for the case of a Lie algebra of skew-symmetric operators on a Hilbert space $H$.

An operator $X$ will be called a pregenerator on $D$ in case it has a closure $\bar{X}$ generating a strongly continuous one parameter group of operators in the sense of Hille and Phillips [6] (denoted by $U[t, X]$ here). Sufficient conditions for this are given in [6]. A counter-example of Nelson [8] refutes the natural conjecture that every $C^{\infty} \mathrm{Lie}$ algebra of pregenerators (each individually exponentiable) is exponentiable in the sense discussed above. We give a number of different sufficient conditions for the exponentiability of $C^{\infty}$ Lie algebras of pregenerators on Banach spaces. Various of the results can be extended to suitably defined " $C$ " and " $C$ " Lie algebras.

1 This work was supported in part by the National Science Foundation, both through a Fellowship and through NSF GT 1611. Several of the results obtained form a part of a dissertation submitted to Princeton University in candidacy for the degree of Ph.D. The author extends his thanks to Professor E. Nelson for his many suggestions and encouragement. 
2. Results. We make extensive use of an auxiliary Banach space, $x^{1}$.

Definition. Let $X_{1}, \cdots, X_{n}$ be a basis for the $C^{\infty}$ Lie algebra of pregenerators $(g, D)$. Then on $\bigcap_{i=1}^{n} D\left(\bar{X}_{i}\right)$ we may define

$$
\|f\|_{1}=\|f\|+\sum_{i=1}^{n}\left\|\bar{X}_{i} f\right\| .
$$

The space $X^{1}$ is the closure of $D$ in this norm, as a subset of the obviously complete space $\bigcap_{i=1}^{n} D\left(\bar{X}_{i}\right)$.

It is not difficult to show that $x^{1}$ is independent of the choice of basis; different bases give equivalent 1 -norms for $X^{1}$. More important, each $X \in g$ extends in a natural way to a member of $B\left(x^{1}, x\right)$, the bounded operators from $x^{1}$ to $X$ (we will denote this extension by $X$ also). Since $g$ is finite dimensional, the $B\left(X^{1}, x\right)$ norm, $|\|X \mid\|$, determines the unique usual topology of $g$; this observation is instrumental in several of the proofs discussed below in paragraph 3.

The fundamental result of the theory requires conditions on both the global groups and their infinitesimal pregenerators:

Theorem 1. A $C^{\infty}$ Lie algebra of pregenerators is exponentiable whenever the following three conditions hold:

(A) $U[t, X]$ leaves $x^{1}$ invariant, for all $t \in R$ and $X \in g$,

(B) for $X$ and $Y$ in $g, t \in R$ and $f \in x^{1}$

$$
U[t, X] Y U[-t, X] f=\exp (t \operatorname{ad} X)(Y) f
$$

where ad $X(Y)=X Y-Y X$, and

(C) $\|U[t, X]\|: R \times g \rightarrow R$ is locally bounded at $(0,0)$.

Conditions (A) and (B) are difficult to check in applications; they may be replaced by a more natural pair of the same semi-global type:

TheOREM 2. A $C^{\infty}$ Lie algebra of pregenerators is exponentiable whenever

(A') $U[t, X]$ leaves $D$ invariant, for all $t$ and $X$,

$\left(B^{\prime}\right)$ for $f \in \mathbb{D}$ and any $X$ and $Y$ in $g,\|Y U[t, X] f\|$ is locally bounded at $t=0$, and

(C) $\|U[t, X]\|: R \times g \rightarrow R$ is locally bounded at $(0,0)$.

In the case of a finite dimensional Lie algebra $g$ of $\left(C^{\infty}\right)$ vector fields on a manifold $M$, the invariance condition $\left(\mathrm{A}^{\prime}\right)$ and the weak "smoothness" condition $\left(\mathrm{B}^{\prime}\right)$ are natural, and $(\mathrm{C})$ is automatic; this provides the best example of an application of Theorem 2. More specifically, we regard $g$ to be defined on the natural domain of $D$ of $C^{\infty}$ functions with compact support. $D$ is dense in the Banach algebra 
$x=C_{0}(M)$ of continuous functions vanishing at $\infty$ on $M$. The assumption " $X$ is a pregenerator" means that $X$ generates a one parameter Lie transformation group on $M$ which acts on $C_{0}(M)$ by "translation" to provide a group of isometric automorphisms, $U[t, X]$ on $C_{0}(M)$.

Both invariance of $D$ and the smoothness condition $\left(B^{\prime}\right)$ are easy consequences of the compact support of each $f \in D$ and of Lie's Hauptsatz (local). Theorem 2 then insures that a finite dimensional Lie algebra of "pregenerator" vector fields exponentiates to give a global representation of $G$ as a group of isometric automorphisms of $C_{0}(M)$; this can be shown to arise from an action of $G$ as a Lie transformation group on $M$.

A result of purely infinitesimal type is also useful.

TheOREM 3. $A C^{\infty}$ Lie algebra of pregenerators is exponentiable whenever

$\left(\mathrm{A}^{\prime \prime}-\mathrm{B}^{\prime \prime}\right)$ for each $X \in g$ there exists a $\omega^{1}(X) \geqq 0$ such that when $|\lambda|>\omega^{1}(X),(\lambda-X)$ is dense in $X^{1}$, and

(C) $\|U[t, X]\|: R \times g \rightarrow R$ is locally bounded at $(0,0)$.

If the resolvent $(\lambda-X)^{-1}$ leaves the domain $D$ invariant, for suitably large $|\lambda|$, an important special case of Theorem 3 is obtained (D is dense in $x^{1}$ by construction). The full strength of Theorem 3 is required in order to give an infinitesimal construction of the irreducible unitary representations of classical groups such as the Lorentz group, on an abstract Hilbert space. Every such representation is the direct sum of finite dimensional irreducible representations of a maximal compact subgroup; one chooses an orthonormal basis for the Hilbert space $x$ from suitably chosen bases for these finite dimensional representations. As several authors have shown (e.g.: Bargmann [1], Dixmier [3]) it is then possible to define the entire Lie algebra $g$ for the representation on the set $D$ of finite linear combinations of basis elements so that $(g, D)$ is a $C^{\infty}$ Lie algebra. Proofs that each $X \in g$ so defined is a pregenerator (essentially skew adjoint) are standard, but this also follows from $\left(A^{\prime \prime}-B^{\prime \prime}\right)$ once this is verified. One proceeds by modifying equation (2) in a natural way to convert $X^{1}$ into a Hilbert space, relates the inner product on $X^{1}$ to that of $X$, then employs the commutation properties of $g$ on $D$ to verify that there is no vector in $x^{1}$ orthogonal to $(\lambda-X) D$. The computations involved make this method of infinitesimal construction much more tedious than the application of the theorem of Nelson mentioned above (illustrated in Dixmier [3]).

In both of the examples discussed above, condition (C) follows 
easily from the nature of the groups involved. In general, it is valuable to remove this condition.

THEOREM 4. Let $(g, D)$ be a $C^{\infty}$ Lie algebra of pregenerators. If $(\mathrm{A})-(\mathrm{B}),\left(\mathrm{A}^{\prime}\right)-\left(\mathrm{B}^{\prime}\right)$ or $\left(\mathrm{A}^{\prime \prime}-\mathrm{B}^{\prime \prime}\right)$ hold then $(\mathrm{g}, \mathrm{D})$ is exponentiable and (C) holds automatically.

3. Discussion of proofs. Theorems 2 and 3 are obtained from Theorem 1 by showing that conditions $\left(\mathrm{A}^{\prime}\right)-\left(\mathrm{B}^{\prime}\right)$ imply $A$ and $B$, and similarly that $\left(A^{\prime \prime}-B^{\prime \prime}\right)$ implies $A$ and $B$. The latter implication is established by means of resolvents and Laplace transforms.

Theorems 1 and 4 are proved independently. To establish either of these results, we use the type of argument outlined in Theorem 63 of Pontrjagin [9] to show that we need only obtain a strongly continuous local representation of $G$. This local representation is defined on the domain of $\exp ^{-1}: G \rightarrow g$ by

$$
U(\exp (t X))=U[t, X] .
$$

Three different arguments are available for obtaining this local exponentiability of $g$ from conditions (A) and (B); each requires some special auxiliary assumption as follows:

(1) $g$ is semisimple, or

(2) $g$ is either solvable or the direct sum of an exponentiable ideal and an exponentiable subalgebra, or

(3) $g$ is arbitrary but satisfies condition (C).

Theorem 4 follows from arguments (1) and (2), coupled with Levi's theorem on the decomposition of an arbitrary $g$ into a semisimple subalgebra and a solvable ideal (Jacobson [7, p. 91]).

Argument 3, on the other hand, is quite elementary and avoids all structural considerations. We outline it here, in view of its application to the important cases in the literature mentioned above.

Strong continuity of the representation is obtained as a by-product of the proof that the map defined by (4) is locally an algebraic isomorphism. If we write $Z(X, t Y)=\exp ^{-1}(\exp (X) \exp (t Y))$ wherever the right hand side is defined ( $Z$ can be computed in terms of the structure constants of $g$ by the Campbell-Hausdorff formula), we have the desired isomorphism iff

$$
U[1, X] U[t, Y]=U[1, Z(X, t Y)],
$$

or equivalently, for each vector $f \in \mathfrak{X}$

$$
k(t) f=U[1, X] U[t, Y] U[-1, Z(X, t Y)] f \equiv f .
$$

Equation $\left(5^{\prime}\right)$ is verified for $f$ in dense $x^{1}$ by the simple expedient of 
computing its $t$-derivative and showing that this vanishes identically for $t$ in a suitable range. It is not difficult to show that $k(0) f=f$, so we conclude that $k(t) f \equiv f$ for all $t$ in this range; extension to arbitrary $f$ is immediate.

The verification that $k(t) f$ is differentiable in $t$, with vanishing derivative, is technical. Details will be published elsewhere. Modulo a straightforward verification that the "product rule" applies to the differentiation of $U[t, Y] U[-1, Z(X, t Y)] f$, the proof depends upon a calculation of the derivative

$$
\frac{d}{d t}(U[-1, Z(X, t Y)] f)=F(t, X, Y) U[-1, Z(X, t Y)] f,
$$

where $F: R \times g \times g \rightarrow g$ is determined entirely by the structure constants of $g$ qua abstract Lie algebra. The exact form of equation (6) is obtained by integration from condition (B), using a generalization of a device of Yosida [11]:

$$
U\left[s, Z_{1}\right] f-U\left[s, Z_{2}\right] f=\int_{0}^{\bullet} U\left[r, Z_{1}\right]\left(Z_{1}-Z_{2}\right) U\left[s-r, Z_{2}\right] f d r
$$

valid for $f \in x^{1}$. Strong continuity follows from (7) and (C).

One then shows that the vanishing of $(d / d t) k(t) f$ depends entirely upon whether $Y+F(t, X, Y)$ vanishes; this fact concerns only the structure of $g$. This is verified by the observation that if one begins with a known locally faithful representation of $G$ where $\left(5^{\prime}\right)$ holds, and repeats the argument, the corresponding faithful representative of $Y+F(t, X, Y)$ must vanish. The faithful local finite dimensional representation obtained by exponentiating Ado's faithful finite dimensional representation of $g$ provides a convenient analytic representation for this purpose (cf. Jacobson [7, p. 202]).

\section{REFERENCES}

1. V. Bargmann, Irreducible unitary representations of the Lorentz group, Ann. of Math. 48 (1947), 568-640.

2. P. Cartier and J. Dixmier, Vecteurs analytiques dans les representations des groupes de Lie, Amer. J. Math. 80 (1958), 131-145.

3. J. Dixmier, Representations integrables du groupe de De Sitter, Bull. Soc. Math. France 89 (1961), 9-41.

4. L. Gärding, Note on continuous representations of Lie groups, Proc. Nat. Acad. Sci. U.S.A. 33 (1947), 331-332; see also, Vecteurs analytiques dans les representations des groupes de Lie, Bull. Soc. Math. France 88 (1960), 73-93.

5. Harish-Chandra, Representations of a semi-simple Lie group on a Banach space. I, Trans. Amer. Math. Soc. 75 (1953), 185-243.

6. E. Hille and R. S. Phillips, Functional analysis and semi-groups, Amer. Math. 
Soc. Colloq. Publ. Vol. 31, Amer. Math. Soc., Providence, R. I., rev. ed., 1957.

7. N. Jacobson, Lie algebras, Interscience, New York, 1962.

8. E. Nelson, Analytic vectors, Ann. of Math. 70 (1959), 572-615.

9. L. Pontrjagin, Topological groups, Princeton Univ. Press, Princeton, N. J., 1939.

10. I. E. Segal, $A$ class of operator algebras which are determined by groups, Duke Math. J. 18 (1951), 221-265.

11. S. Yosida, On the differentiability and the representation of one parameter semigroup of linear operators, J. Math. Soc. Japan 1 (1948), 15-21.

PRINCETON UNIVERSITY AND

University of California, Berkeley

\title{
WEAK LEVI CONDITIONS IN SEVERAL COMPLEX VARIABLES ${ }^{1}$
}

\author{
BY AVNER FRIEDMAN
}

Communicated by R. P. Boas, June 28, 1965

1. Introduction. Let $\Omega=\left\{z ; z \in \Omega_{0}, \rho(z)<0\right\}$ be a bounded domain in $C^{n}$, where $\rho \in C^{2}\left(\Omega_{0}\right), \Omega_{0}$ a neighborhood of $\Omega$, and let grad $\rho \neq 0$ on $\partial \Omega$. As is well known, if $\Omega$ is a domain of holomorphy then for any $x^{0} \in \partial \Omega$,

(1) $L\left(\rho\left(x^{0}\right), w\right) \equiv \sum_{j, k=1}^{n} \frac{\partial^{2} \rho\left(x^{0}\right)}{\partial z_{j} \partial \bar{z}_{k}} w_{j} \bar{w}_{k} \geqq 0 \quad$ whenever $\sum_{j=1}^{n} \frac{\partial \rho\left(x^{0}\right)}{\partial z_{j}} w_{j}=0$,

and, if (1) holds with strict inequality (for $w \neq 0$ ) then $\Omega$ is a domain of holomorphy. (1) is called the Levi condition (LC) and, in case of strict inequality, the strict LC. One of the consequences of the present work is that the above statement remains true if the assumption $\rho \in C^{2}$ is replaced by $\rho \in H^{2, \infty}$ (see $\$ 2$ ).

In what follows $\Omega$ is always given by $\rho$ as above, where $\rho \in C^{1}\left(\Omega_{0}\right)$, $\operatorname{grad} \rho \neq 0$ on $\partial \Omega$.

2. Definitions. If $\rho$ has second weak derivatives which belong to $L^{p}\left(\Omega_{0}\right)(1<p<\infty)$ then we say that $\Omega$ and $\rho$ belong to $H^{2, p}$. Actually we shall only need the derivatives $\partial^{2} \rho / \partial z_{j} \partial \bar{z}_{k}$ to belong to $L^{p}$, but then

1 This work was partially supported by the Alfred P. Sloan Foundation and by Nasa Grant NGR 14-007-021. 